\title{
La atribución de responsabilidades políticas en Estados descentralizados*
}

\author{
Santiago Lago Peñas \\ REDE, IEB y Universidade de Vigo \\ Ignacio Lago Peñas \\ Universitat Pompeu Fabra de Barcelona
}

\section{Resumen}

En este artículo se estudian con datos de encuesta los determinantes institucionales e individuales de la atribución correcta de responsabilidades políticas en el Estado de las Autonomías en España, así como el proceso de aprendizaje de los individuos a lo largo del tiempo. Nuestros resultados muestran que la atribución de las competencias es más acertada cuando están exclusivamente en manos del Gobierno central, en las llamadas Comunidades Autónomas de «vía rápida» y a medida que se avanza en el proceso de descentralización. Finalmente, se plantean dos hipótesis, la de la información y la del interés, para explicar las diferencias entre los ciudadanos. Los resultados muestran que el impacto de las variables individuales, como la edad o el nivel de estudios, depende de la política que se estudie.

Palabras clave: descentralización, comunidades autónomas, España, gobierno, responsabilidades.

Clasificación JEL: D72.

\begin{abstract}
In this paper we rely on individual data from Spain to show the individual and institutional determinants of responsibility attribution and to what extent individuals are able to learn about responsibilities. Our results show that the attribution of responsibility is more accurate when the power is exclusively in the hands of the national government, in the 'fast-track' Regions and as the decentralization process goes by. Finally, we propose the information and the self-interest hypotheses to account for differences among citizens. Our findings show that the impact of individual variables, such as age or education, differs across policy areas.
\end{abstract}

Keywords: decentralization, autonomous communities, Spain, government, responsibility.

JEL classification: D72.

* Nos gustaría agradecer la financiación del Ministerio de Ciencia e Innovación (ECO2010-15553 y CSO2010-16319). Algunos de los datos empleados en el artículo proceden del proyecto Making Electoral Democracy Work (véase BLAIS, 2010). Este artículo actualiza y extiende trabajos previos de ambos autores; en particular, LAGO PEÑAS y LAGO PEÑAS (2010 y 2011). Los comentarios de un evaluador anónimo han sido muy útiles para revisar el manuscrito. 


\section{Introducción}

Uno de los argumentos más conocidos en las teorías analíticas de la democracia (Przeworski et al., 1999) es que el control electoral de los gobiernos, y por extensión su carácter representativo, depende de que los votantes atribuyan correctamente las responsabilidades políticas. Si los votantes tienen un velo de ignorancia sobre el grado de responsabilidad que cabe atribuir a un gobierno en los resultados económicos (buenos o malos), el voto retrospectivo no garantiza que sean castigados los malos gobiernos y recompensados los buenos. Y dados los incentivos que tienen los malos políticos para hacerse pasar por buenos, las declaraciones de los gobiernos son un cheap-talk. Como concluye Rudolph (2003b: 700), el concepto de responsabilidad está en el corazón de las teorías del control electoral en las democracias.

En los Estados centralizados en los que existe un solo nivel de gobierno, sobre todo cuando las relaciones económicas internacionales son limitadas, la atribución de responsabilidades es relativamente sencilla: todo está en manos del gobierno central $^{1}$. Sin embargo, cuando se descentraliza el poder dentro del Estado y/o tie-nen lugar fuertes procesos de integración económica y política en el plano inter-nacional, se dificulta que los ciudadanos puedan imputar responsabilidades políticas y, por tanto, controlen adecuadamente a los gobiernos (Lago Peñas y Lago Peñas, 2011).

En las últimas décadas se observa tanto una tendencia global hacia la descentralización, definida como la transferencia de poder político y fiscal autónomos a los gobiernos subnacionales (O'Neill, 2003: 1070), como una creciente integración supranacional. A diferencia de lo que sucedía en los primeros años setenta del siglo pasado, cuando la mayoría de los países respondían al modelo de Estado-nación centralizado, en estos momentos «alrededor del 95 por 100 de las democracias [...] han elegido gobiernos subnacionales y países en todas las partes del mundo -grandes y pequeños, ricos y pobres- están transfiriendo poderes políticos, fiscales y administrativos a los niveles de gobierno subnacionales» (Banco Mundial, 2000: 107). Al mismo tiempo, si los Estados ceden la decisión sobre determinadas políticas a organizaciones supranacionales, la responsabilidad pasa a ser compartida. Por ejemplo, poco o nada tienen que ver los Estados individuales con la política monetaria o la política exterior de la Unión Europea, de modo que los ciudadanos no deberían castigar o recompensar a los gobiernos nacionales por los resultados que se consigan en ambas áreas.

En este artículo estudiamos cómo afecta la descentralización política y económica, el más general de los procesos señalados anteriormente, a la atribución de responsabilidades por las acciones de gobierno. En concreto, exploramos dos argumentos. En primer lugar, en qué medida el ritmo y el tipo de descentralización dentro de los

${ }^{1}$ El gobierno, por supuesto, puede tratar de evitar la atribución de responsabilidad por un resultado negativo mediante las justificaciones (esto es, se acepta la responsabilidad, pero se niega que las consecuencias de las decisiones sean negativas) o las excusas basadas en circunstancias mitigantes que tengan que ver con el pasado (por ejemplo, las políticas del gobierno anterior) o el presente (por ejemplo, un nuevo escenario económico: McGRAW, 1990). 
Estados distorsionan las percepciones de los ciudadanos sobre los niveles de gobiernos competentes en las políticas y si los ciudadanos aprenden a lo largo del tiempo. En segundo lugar, cuáles son los determinantes de las diferencias en la capacidad de los ciudadanos para atribuir correctamente las responsabilidades políticas. El análisis empírico se centra en España. El notable proceso de descentralización política y económica en tres décadas, que han llevado a un Estado unitario y centralizado en los años setenta del siglo pasado a ser uno de los países más descentralizados del mundo en lo que atañe al gasto público, y las asimetrías entre las Comunidades Autónomas, hacen de nuestro país un caso de estudio del mayor interés. Además de revisar la investigación existente, mostramos algunos problemas metodológicos de la literatura sobre el tema, en particular la utilización de índices que agregan la atribución correcta de responsabilidades en distintos temas.

Nuestros principales hallazgos apuntan, en términos institucionales, que la atribución de responsabilidades en España es mejor cuando las competencias están en manos del gobierno nacional y en las Comunidades que más tiempo han disfrutado de competencias propias. En cuanto a los determinantes individuales, la principal conclusión es que el impacto de las variables depende de la política que se estudia; la edad es en cualquier caso la que tiene un efecto más sistemático. Este resultado significa que la utilización de índices que suman el conocimiento de la responsabilidad en diferentes políticas es problemática.

El resto del trabajo se organiza de la siguiente manera. En el segundo apartado se discuten los argumentos que explican el impacto de la descentralización sobre la atribución de responsabilidades políticas entre los niveles de gobierno. A continuación se hace una breve descripción del proceso de descentralización en España. El análisis empírico de las secciones cuarta y quinta se detiene, por un lado, en las diferencias en la asignación correcta de responsabilidades a lo largo del tiempo y entre las Comunidades Autónomas y, por otro, en los determinantes de las diferencias entre ciuddadanos. Para lo primero utilizamos datos para el conjunto de las Comunidades Autónomas procedentes de barómetros del Instituto de Estudios Fiscales. Para lo segundo, estudiamos exclusivamente Cataluña, en particular las elecciones autonómicas de 2012, para la que disponemos de una encuesta de panel especialmente apropiada para nuestros propósitos y elaborada por el proyecto $\mathrm{Ma}$ king Electoral Democracy Work. El artículo termina con las conclusiones y algunas reflexiones sobre los aspectos de la descentralización que se deben estudiar con más detalle.

\section{La atribución de responsabilidades políticas en Estados descentralizados}

La información sobre las competencias de los distintos niveles de gobierno no se distribuye aleatoriamente entre los ciudadanos, sino que existen variables individuales e institucionales que explican las diferencias entre ellos. En primer lugar, 
se puede asumir que el conocimiento de las responsabilidades de los gobiernos es simplemente una expresión de la información política que poseen los ciudadanos, de modo que no habría diferencias entre este tema, las evaluaciones correctas de la economía o el conocimiento de los nombres de políticos relevantes. La investigación sobre cuánto saben los votantes sobre economía, por ejemplo, muestra que no están demasiado al corriente de los datos sobre desempleo, inflación, crecimiento o deuda pública (Aidt, 2000: 357-359). En general, son los individuos de cierta edad, con mayor nivel de estudios y una renta por encima de la media los que están mejor informados. En este mismo sentido, Duch et al. (2000) concluyen que (la heterogeneidad en) las evaluaciones de la economía dependen de la cercanía al partido en el gobierno, las situaciones económicas personales y la sofisticación e información de los votantes.

Si nos detenemos en la investigación sobre los determinantes individuales de las atribuciones de responsabilidades, la ideología y la identificación de partido son especialmente importantes de acuerdo con Rudolph (2003a, 2003b). No obstante, son los recientes estudios de León $(2010,2012)$, precisamente sobre el Estado de las Autonomías en España, los que tratan directamente la atribución de responsabilidades en Estados descentralizados. En su trabajo de 2010, León concluye que la educación y los niveles declarados de información son las variables que más influyen en un índice de 0 a 5 que mide si los encuestados identifican correctamente qué nivel de gobierno (local, regional o central) tiene la responsabilidad principal sobre cinco políticas: sanidad, educación, vivienda, empleo e industria y comercio. Además, la participación electoral, el interés por la política nacional y sentirse más español que de la Comunidad Autónoma se correlacionan significativamente con el índice (las dos primeras variables de un modo positivo, la segunda negativamente); mientras que el interés en la política regional, haber nacido en la Comunidad Autónoma, la ocupación o la edad no influyen.

Sin embargo, en un artículo posterior, en el que la variable dependiente es un índice similar que oscila de 0 a 4, las conclusiones de León (2012) son algo distintas: sentirse más español que de la Comunidad Autónoma y solo algunas categorías en educación influyen significativamente en la atribución correcta de responsabilidades. La participación electoral pasa ahora a ser irrelevante. No hay ninguna variable en el modelo que mida el grado de información de los individuos.

En segundo lugar, la literatura sobre comportamiento político ha girado en los últimos años hacia el institucionalismo y plantea que los arreglos institucionales influyen en el grado de claridad de las responsabilidades políticas. Según Powell y Whitten (1993), hay cinco características institucionales que complican la atribución correcta de responsabilidades. Se trata de (i) muchos partidos en el gobierno, (ii) la existencia de gobiernos minoritarios, (iii) partidos débilmente cohesionados, (iv) que alguna institución que participa en el desarrollo de las políticas esté en manos de los partidos de la oposición, y (v) comisiones legislativas fuertes presididas por miembros de los partidos de la oposición. El mecanismo causal detrás de estas variables es que la capacidad de los votantes para asignar responsabilidades 
políticas es una función de la facilidad con la que puedan identificar a la persona competente. Cuanto mayor es la percepción de que el control único de las políticas están en manos del gobierno, más probable es que se le asigne la responsabilidad por los resultados económicos y políticos que se alcanzan (Powell y Whitten, 1993: 398).

Esta discusión de la claridad de las responsabilidades se centra en su dimensión horizontal, esto es, en el grado de responsabilidad dentro de un gobierno nacional. En los sistemas de gobierno multinivel, en los que se produce una dispersión de la autoridad política en múltiples niveles de gobierno, aparece también una dimensión vertical de la responsabilidad. De acuerdo con Anderson (2006: 450) y Cutler (2004: 19-22), hay dos razones para que los gobiernos multinivel debiliten la claridad de responsabilidades. La primera consiste en que los votantes necesitan más información para saber cuáles son los resultados de las actuaciones de cada nivel de gobierno y la responsabilidad de cada uno de ellos. Cuando solo hay un gobierno (nacional), (casi) toda la responsabilidad es suya. Pero si hay también gobiernos subnacionales, la asignación de responsabilidades ya no puede ser directa: los votantes tienen que saber ahora si las competencias sobre cada tema son compartidas o exclusivas. Y la segunda es que, cuando se superponen niveles de gobierno, los políticos tienen fuertes incentivos para apropiarse de la responsabilidad por los buenos resultados políticos o económicos y eludirla cuando estos resultados son malos.

De nuevo, los trabajos de León $(2010,2012)$ permiten dar un paso adelante y aclarar qué tipos de Estados descentralizados son mejores o peores para que los ciudadanos sepan determinar qué gobierno es el responsable de cada política. A partir de datos individuales sobre el funcionamiento del Estado de las Autonomías en España, la autora concluye que los ciudadanos son más capaces de atribuir correctamente las responsabilidades políticas cuando el control del gasto y los impuestos se concentra fundamentalmente en un único nivel de gobierno, ya sea el nacional o el regional. Se trata, en definitiva, del argumento de Powell y Whitten que veíamos antes, pero aplicado a Estados descentralizados. Además, León (2012) apunta que el proceso de aprendizaje de los ciudadanos sobre la distribución de competencia está sesgado, puesto que sobrevaloran los poderes de los gobiernos regionales.

\section{La descentralización en España}

La Constitución española (CE) aprobada en 1978 transformó radicalmente la estructura política y administrativa del Estado. De un modelo unitario y fuertemente centralizado se pasa a otro en el que las responsabilidades sobre el gasto se hallan muy descentralizadas. Desde un valor nulo a finales de los años setenta, las CCAA 
llegan a gestionar el 39,4 por 100 del gasto público total en $2011^{2}$. En la actualidad, las CCAA tienen en sus manos los principales servicios públicos en materia social, con una administración central que, en este ámbito, solo se reserva competencias sobre la legislación básica que regula sanidad, servicios sociales, vivienda o educación y las transferencias canalizadas a través de la Seguridad Social.

Esta fortísima descentralización política y financiera en España se caracteriza por una triple asimetría, que afecta tanto a las competencias como a los ingresos tributarios. La primera asimetría se refiere al ritmo de cesión de poderes a los diferentes territorios. Aunque a la hora de hablar de la velocidad de transferencia de responsabilidades de gasto se suele distinguir entre las Comunidades de «vía lenta» (o del artículo 143 de la CE) y «las de vía rápida» ${ }^{3}$, la realidad es que la descentralización ha trascendido el doble menú para situarse próxima a un proceso a la carta.

El proceso de descentralización no ha avanzado de forma regular en el tiempo, sino que presenta puntos de inflexión vinculados al devenir del proceso de cesión de materias (Gil-Ruiz e Iglesias, 2007). En los años ochenta se produce el grueso de traspasos, a excepción de las (importantes) competencias sanitarias y educativas en el caso de las Comunidades Autónomas del artículo 143. Esto se traduce en porcentajes alrededor del 20 por 100 de gasto público (véase el Gráfico 1). En la década de los noventa se siguen produciendo traspasos, pero con una incidencia presupuestaria inferior, de forma que las Comunidades Autónomas estabilizan su participación en el gasto en el entorno del 25 por 100. A partir de 1999 se produce un segundo gran impulso descentralizador en términos globales, con la concreción del traspaso de la educación y sanidad (a partir de 2002) a las Comunidades Autónomas que todavía no tenían asumidas estas competencias; para pasar a tener prácticamente las mismas competencias que las demás.

La segunda de las asimetrías se manifiesta en los modelos de financiación de esas competencias. Más allá de la bien conocida distinción entre el modelo común y el modelo foral de Navarra y País $\mathrm{Vasco}^{4}$, hay que referirse al modelo canario (integrado dentro del común ${ }^{5}$ ), las particularidades de las Comunidades Autónomas uniprovinciales, y las diferencias que existieron hasta 2002 entre las Comunidades del artículo 143 y las del 151 y asimiladas.

Finalmente, existe una dinámica diferencial del gasto respecto a los ingresos autónomos. La descentralización del sistema fiscal español en las CCAA de régimen

${ }^{2}$ Según los datos incluidos por el Ministerio de Hacienda y Administraciones Públicas en la Actualización del programa de estabilidad del Reino de España. 2012-2015 (http://serviciosweb.meh. es/apps/dgpe/TEXTOS/progest/progest.pdf), las CCAA habrían gastado en 2011 el equivalente al 17,2 por 100 del PIB español sobre un gasto público global equivalente al 43,6 por 100 .

${ }^{3}$ Los términos «vía rápida» y «vía lenta» hacen referencia a la vía legal a través de la que se obtiene la autonomía, que tiene incidencia muy importante sobre la velocidad de traspaso de competencias.

${ }^{4}$ En esencia, Navarra y las Diputaciones Forales vascas recaudan la práctica totalidad de los tributos en sus territorios. La recaudación obtenida sirve para financiar las competencias autonómicas y para contribuir a las competencias no asumidas a través del cupo vasco y la aportación navarra.

${ }^{5}$ Véase al respecto el trabajo de FERNÁNDEZ LLERA y LAGO PEÑAS (2011). 
común ha sido prácticamente nula hasta $1997^{6}$. Entre las consecuencias negativas de ello, aparece la falta de responsabilidad fiscal de los gobiernos autonómicos. La revisión del sistema que entra en vigor en 1997 y, en mayor medida, las que se aprueban en 2001 y 2009 han venido a corregir solo parcialmente esta deficiencia ${ }^{7}$. Esa fuerte dependencia de los recursos transferidos ha provocado que el gasto de cada Comunidad Autónoma haya evolucionado al compás marcado por el proceso de traspaso de competencias y la dinámica de los recursos transferidos.

\section{GRÁFICO 1}

DESCENTRALIZACIÓN DEL GASTO PÚBLICO (1988-2005) (estructura porcentual)

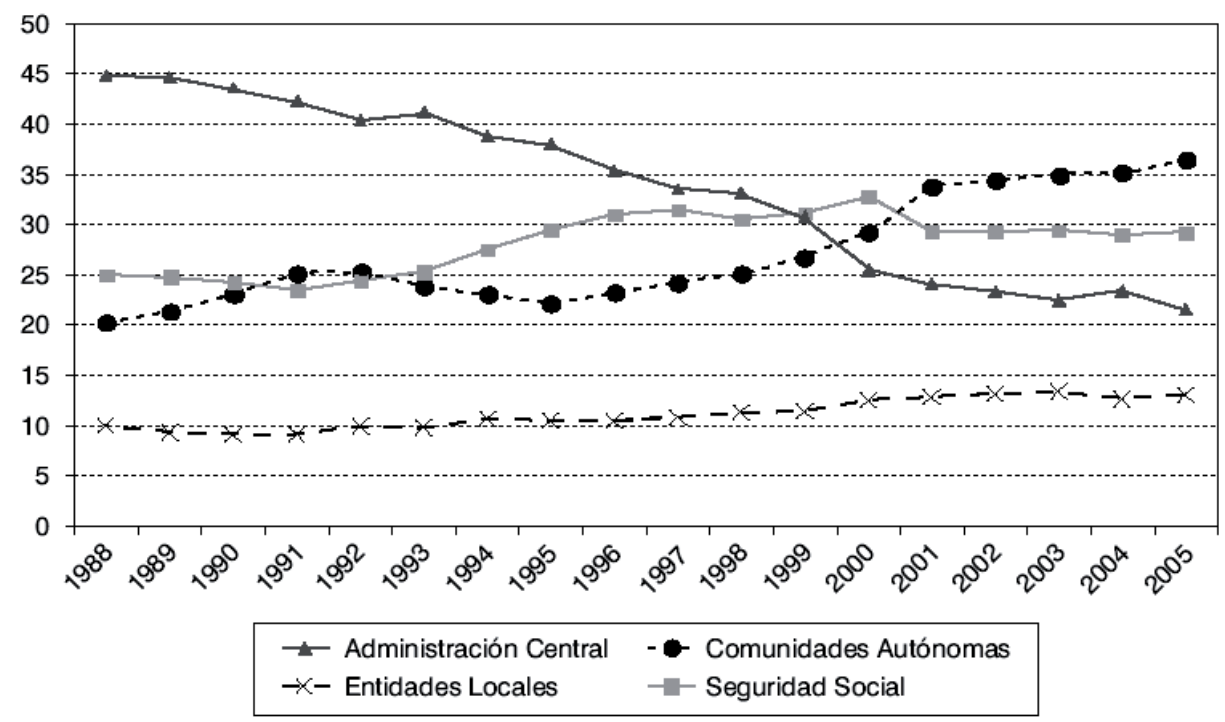

FUENTE: GIL-RUIZ e IGLESIAS (2007).

${ }^{6}$ Hasta esa fecha estuvieron vigentes el sistema para el quinquenio 1987-1991 y para el quinquenio 1992-1996. Antes de 1987 la financiación de las CCAA carecía de un modelo stricto sensu.

${ }^{7}$ Sobre la problemática de la descentralización tributaria y las últimas reformas del sistema pueden consultarse los libros colectivos coordinados por LAGO PEÑAS y MARTÍNEZ-VÁZQUEZ (2009 y 2011). 


\section{Los determinantes institucionales y el aprendizaje en la asignación de responsabilidades}

El objetivo de este apartado es aproximarnos a las diferencias en el tiempo y entre las Comunidades Autónomas en el grado de conocimiento que tienen los individuos sobre las responsabilidades de gasto en España. Para ello es particularmente útil el módulo rotatorio incluido en la encuesta sobre «Opiniones y actitudes fiscales de los españoles en 2005» elaborada por el Instituto de Estudios Fiscales (IEF, 2006) ${ }^{8}$. Primero, porque en el cuestionario se incluye una batería de preguntas sobre la responsabilidad política en distintos servicios públicos y prestaciones sociales; entre ellos los cuatro que nos interesan en este trabajo. Y segundo, porque la encuesta se realiza en 2005, cuando ya existe homogeneidad competencial en sanidad y educación, pero aun ha transcurrido relativamente poco tiempo desde que el proceso ha culminado en las CCAA de «vía lenta». Por ello, es posible que sigamos observando diferencias significativas en el aprendizaje respecto a quienes cuentan con responsabilidades en sanidad y educación desde hace mucho antes.

Puesto que la legislación estatal básica en sanidad y educación sigue en manos de la administración central, cuando en la encuesta del IEF se pregunta por la administración responsable, hemos considerado como correctas las respuestas que asignan la responsabilidad a las Comunidades o las que señalan que está compartida entre las Comunidades y el Estado. Pese a todo, como se puede observar en el Cuadro 1, más de la mitad de los encuestados, el 53 por 100 en la sanidad y el 54 por 100 en la educación, asignan incorrectamente las responsabilidades. Asignación que tiende a recaer en el gobierno central. Por su parte, cuando nos referimos a prestaciones integradas en el sistema de Seguridad Social, diseñado y ejecutado de forma centralizada bajo el principio de «caja única», un porcentaje muy inferior de ciudadanos atribuye incorrectamente las responsabilidades de gasto: un 30 por 100 en el caso de las pensiones de jubilación y un 26 por 100 en el del seguro por desempleo. Es decir, cuando las competencias las detenta el gobierno nacional, los ciudadanos son más capaces de imputar correctamente las responsabilidades políticas.

${ }^{8}$ Datos de la encuesta: Ámbito geográfico, Territorio nacional. Población, Universo total de individuos de 18 y más años, económicamente activos e inactivos. Metodología Cuantitativa, mediante aplicación de cuestionario estructurado en hogar. Diseño muestral, Muestreo por conglomerados polietápico con estratificación y subestratificación de las unidades de primera etapa (secciones censales). Tamaño muestral, 1.410 entrevistas personales en hogar. Afijación no proporcional. Margen de error $\pm 2,7$ para los datos globales en el supuesto de muestreo aleatorio simple y categorías equiprobables. $(\mathrm{p}=\mathrm{q}=50$ por ciento) a un nivel de confianza del 95 por ciento. Trabajo de campo, 28 de noviembre a 22 de diciembre de 2005. Empresa adjudicataria, CUANTER, SA. 


\section{CUADRO 1}

«DE LOS SIGUIENTES SERVICIOS PÚBLICOS Y PRESTACIONES SOCIALES, ¿QUIÉN CREE USTED QUE ES EL RESPONSABLE, POR EJEMPLO A LA HORA DE PONER UNA RECLAMACIÓN, EL ESTADO CENTRAL, LA COMUNIDAD AUTÓNOMA, EL AYUNTAMIENTO, O ES COMPARTIDA?»

(\% de respuestas)

\begin{tabular}{|l|c|c|c|c|c|c|}
\hline & \multicolumn{5}{|c|}{ Administración responsable en la actualidad* } \\
\hline Servicios y prestaciones & Estado & $\begin{array}{c}\text { Auto- } \\
\text { nómica }\end{array}$ & Local & $\begin{array}{c}\text { Com- } \\
\text { partida }\end{array}$ & NS/NC & Error \\
\hline Sanidad & 44 & 39 & 3 & 8 & 6 & 53 \\
\hline Educación & 42 & 36 & 6 & 10 & 6 & 54 \\
\hline Pensiones de jubilación & 70 & 16 & 2 & 5 & 7 & 30 \\
\hline Seguro de desempleo & 64 & 20 & 3 & 6 & 7 & 36 \\
\hline
\end{tabular}

FUENTE: IEF (2006).

NOTA: * Excluida la última columna, en negrita, los porcentajes se han calculado sobre los totales de fila. La columna de los errores suma el porcentaje de encuestados que se equivoca.

Puesto que la asignación ya manejada en Lago Peñas y Lago Peñas (2010) de responsabilidades de gasto ha cambiado en el tiempo en España, es posible que si examinamos las Comunidades Autónomas del artículo 151, las asimiladas y las forales, los aciertos sean mayores. Estas Comunidades recibieron los traspasos en materia educativa y sanitaria en los años ochenta y primeros noventa. En efecto, el Cuadro 2 muestra que los errores en la atribución de responsabilidades alcanzan el 43 por 100 en las Comunidades Autónomas del artículo 151, asimiladas y forales, frente al 69 por 100 en las restantes Comunidades; esto es, la diferencia asciende a 26 puntos porcentuales más. Otro tanto sucede en educación, donde los porcentajes de error ascienden al 67 y 46 por 100, respectivamente: 21 puntos porcentuales de diferencia. Se trata de diferencias estadísticamente significativas al 1 por 100 .

\section{CUADRO 2}

ERRORES EN LA ASIGNACIÓN DE RESPONSABILIDADES EN MATERIA EDUCATIVA O SANITARIA

(\% de respuestas)*

\begin{tabular}{|l|c|c|}
\hline & En CCAA, artículo 143 & $\begin{array}{c}\text { En CCAA, artículo 151 y asimiladas } \\
\text { y forales }\end{array}$ \\
\hline Sanidad & 69 & 43 \\
& $(569)$ & $(839)$ \\
\hline Educación & 67 & 46 \\
& $(569)$ & $(839)$ \\
\hline
\end{tabular}

FUENTE: IEF (2006).

NOTA: * Entre paréntesis, el número de observaciones. 
Por el contrario, los errores en la asignación de responsabilidades son menores en las Comunidades Autónomas de «vía lenta» cuando se trata de competencias centralizadas. Así, según el Cuadro 3, las respuestas incorrectas se sitúan en el 19 por 100 tanto en el caso de las pensiones de jubilación como en el del seguro por desempleo. Los porcentajes correspondientes al resto de Comunidades se sitúan entre el 38 por 100 y el 44 por 100. En definitiva, estos resultados son consistentes con la evidencia que presenta León (2012).

\section{CUADRO 3}

ERRORES EN LA ASIGNACIÓN DE RESPONSABILIDADES EN MATERIA DE PENSIONES DE JUBILACIÓN, SEGURO DE DESEMPLEO Y PENSIONES DE ENFERMEDAD O INVALIDEZ

( $\%$ de respuestas)

\begin{tabular}{|l|c|c|}
\hline & En CCAA, artículo 143 & $\begin{array}{c}\text { En CCAA, artículo 151 } \\
\text { y asimiladas y forales }\end{array}$ \\
\hline Pensiones de jubilación & 19 & 38 \\
& $(569)$ & $(839)$ \\
\hline Seguro de desempleo & 19 & 44 \\
& $(569$ & $(839)$ \\
\hline
\end{tabular}

FUENTE: IEF (2006).

NOTA: * Entre paréntesis, el número de observaciones.

La información anterior puede complementarse con la edición del barómetro fiscal de 2010 (IEF, 2011), que ofrece datos sobre la atribución de responsabilidades para el período 2005-2010. En el Gráfico 2 se presenta el porcentaje de individuos que asigna al Gobierno central las responsabilidades en pensiones y prestaciones por desempleo y a los gobiernos autonómicos la sanidad y educación. Excluidos los años 2008 y 2009, para los cuales no hay datos, la principal conclusión es que no hay una tendencia definida. Si entre 2005 y 2007 caen las respuestas correctas, en 2010 aumenta sensiblemente el porcentaje de individuos que asigna adecuadamente las responsabilidades. A la espera de disponer de los datos para 2011 y 2012, una posible explicación para esta pauta es que la intensa discusión pública que ha acompañado los recortes en el gasto público enmarcados en el actual proceso de consolidación fiscal habría llevado, de forma no intencionada, a un mejor conocimiento de nuestro Estado de las Autonomías por parte de los ciudadanos. 


\section{GRÁFICO 2}

\section{PORCENTAJE DE ENCUESTADOS QUE ASIGNAN CORRECTAMENTE LAS COMPETENCIAS EN PENSIONES, PRESTACIONES POR DESEMPLEO, EDUCACIÓN Y SANIDAD EN ESPAÑA, 2005-2010}

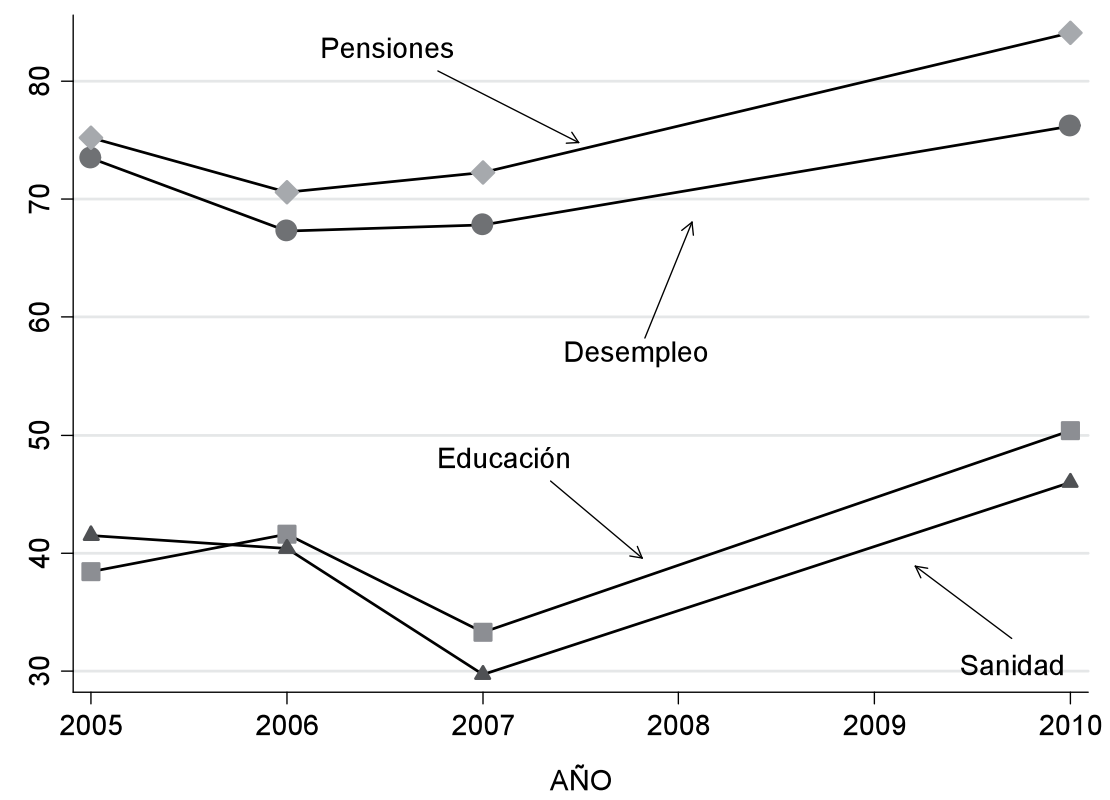

FUENTE: Elaboración propia a partir de IEF (2011).

\section{Los determinantes individuales de la asignación de responsabilidades}

En esta sección del artículo se estudian las variables individuales que explican que algunos ciudadanos conozcan mejor que otros la distribución de competencias entre los niveles de gobierno. Es decir, si antes explicábamos la variabilidad espacial y temporal en la atribución correcta de responsabilidades políticas, ahora nos detenemos en la variabilidad individual.

Al igual que las evaluaciones de la situación económica (Duch et al., 2000), nuestro argumento es que las diferencias entre ciudadanos no son exclusivamente aleatorias, sino sistemáticas. En términos generales, hay dos grandes tipos de factores que dan cuentan de esta variabilidad: la información y la exposición a los medios de comunicación y las características socioeconómicas. En primer lugar, los individuos con más información y, en general, con recursos cognitivos más elevados, esto, es los que tienen más interés por la política, un nivel educativo más alto o simplemente más edad, deberían estar más al corriente de cómo se distribuyen las competencias entre los niveles de gobierno. Se trata de la hipótesis de la información. 
En segundo lugar, nuestra expectativa es que los individuos que más beneficios obtienen de estar informados acerca de la distribución de competencias harán una asignación más precisa de responsabilidades políticas. En otras palabras, el conocimiento de qué nivel de gobierno es competente sobre un determinado tema debería estar correlacionado con la situación socioeconómica de los individuos. Por ejemplo, los jubilados deberían conocer mejor quién decide sobre las pensiones o la sanidad que el resto de ciudadanos; los parados (o trabajadores con mayor riesgo de perder su trabajo) deberían prestar más atención a las cuestiones acerca de las prestaciones por desempleo y los ciudadanos con mayor renta, menos, y los más jóvenes, los estudiantes, deberían seguir en mayor medida las decisiones sobre las políticas de educación. Se trata de la hipótesis del interés.

Esta distinción entre los dos tipos de factores es crucial también en términos metodológicos y empíricos. La literatura existente (León, 2010 y 2012, por ejemplo) ha obviado la hipótesis del interés y, en consecuencia, ha incurrido en un importante sesgo en la estimación del efecto de las variables individuales. La estrategia habitual consiste en usar datos de encuesta para crear un índice que suma el número de temas en los que el encuestado atribuye correctamente la responsabilidad política, sin establecer ningún tipo de ponderación.

Si se pregunta sobre pensiones, sanidad, educación y prestaciones por desempleo, el índice oscilaría entre 4, cuando se acierta qué nivel de gobierno es el responsable de los cuatro temas, y 0 , cuando se yerra en los cuatro temas. De acuerdo con la hipótesis de la información, la edad, por ejemplo, se debería correlacionar positivamente y con la misma intensidad con la atribución de responsabilidades en los cuatro temas. Sin embargo, según la hipótesis de la información, la edad se correlacionaría más positivamente con las responsabilidades en pensiones y sanidad que en prestaciones por desempleo o educación; en estos dos últimos casos, sobre todo en el segundo, la correlación sería incluso negativa. La agregación del impacto que se deriva de las dos hipótesis, y que no siempre van en la misma dirección para todos los temas, convierte el índice en una falacia ecológica.

El propósito del análisis empírico que sigue es doble. Por un lado, determinar cuál es el grado de conocimiento actual de los ciudadanos sobre la distribución de competencias en España, en particular en Cataluña en 2012. Por otro, explicar sus determinantes y mostrar su distinto efecto en función de cuál sea la política que se estudia. Los datos que empleamos proceden de una encuesta de panel en Cataluña con motivo de las elecciones autonómicas del 25 noviembre de 2012. Se trata de una encuesta realizada a través de internet a una muestra representativa de 993 personas y realizada por el proyecto Making Electoral Democracy Work (MEDW $)^{9}$.

${ }^{9}$ El trabajo de campo de la encuesta preelectoral se realizó entre el 16 y 23 de noviembre y el de la postelectoral entre el 26 de noviembre y el 10 de diciembre. Las encuestas fueron realizadas por la empresa canadiense Harris Decima a partir de su propio panel de individuos. Se trata de una muestra estratificada con cuotas por edad, género y nivel educativo. Para más detalles, véase http://electoraldemocracy.com. 
El cuestionario contiene cuatro preguntas acerca de la distribución de competencias entre el Gobierno Central y la Generalitat de Cataluña particularmente apropiadas para estudiar el tema, además de cuestiones sobre sus determinantes en los términos señalados más $\operatorname{arriba}^{10}$. La redacción es la siguiente:

Por favor, indique qué nivel de gobierno tiene la responsabilidad principal en la gestión de las siguientes políticas:

\begin{tabular}{|l|c|c|c|}
\hline & $\begin{array}{c}\text { Gobierno } \\
\text { Central }\end{array}$ & $\begin{array}{c}\text { Generalitat de } \\
\text { Cataluña }\end{array}$ & No sabe \\
\hline Pensiones (1) & - & - & - \\
\hline Sanidad (2) & - & - & - \\
\hline Educación (3) & - & - & - \\
\hline Prestación por desempleo (4) & - & - & \\
\hline
\end{tabular}

La variable dependiente del modelo recoge si los individuos aciertan qué nivel de gobierno es el responsable principal de cada política (valor 1) frente a si no aciertan o no lo saben (valor 0). Para mostrar los problemas que se derivan de usar un índice agregado, en nuestros modelos tendremos cinco definiciones de la variable dependiente: una para cada una de las cuatro políticas, y que puede adoptar el valor 1 o el 0 , y un índice que suma las repuestas correctas y que varía entre 4 , cuando el encuestado asigna correctamente la responsabilidad en las cuatro políticas y 0 , cuando no acierta en ninguno de los casos. Además, hay una sexta variable dependiente, el conocimiento de la existencia de una barrera legal en el sistema electoral de Cataluña, que introducimos en la medida en que solo depende de la hipótesis de la información. De este modo tendremos pistas adicionales sobre el alcance de la hipótesis del interés. La redacción de la pregunta es la siguiente:

Indique, por favor, si la siguiente afirmación es verdadera o es falsa: En su circunscripción, un partido necesita obtener por lo menos el 3\% de los votos para entrar en el Parlament de Cataluña.

$\square$ Verdadero
$\square$ Falso
$\square$ No sabe

${ }^{10}$ Desafortunadamente, no hay ninguna pregunta sobre la ocupación de los encuestados. 
En el Cuadro 4 se muestra el grado de conocimiento de los catalanes sobre la distribución de competencias en España. Como se puede ver, el 86 por 100 de los encuestados acierta cuando se le pregunta qué gobierno tiene la responsabilidad principal sobre las pensiones, el Gobierno central. Los porcentajes son más bajos en el caso de las prestaciones por desempleo, el 81 por 100, sanidad, 70 por 100, y educación, 68 por 100. Estos valores son sensiblemente más altos que cuando se pregunta sobre la barrera legal; de acuerdo con la evidencia que se presenta en el Cuadro 2, apenas en 28 por 100 de los catalanes sabe que hay que sumar al menos el 3 por 100 de los votos (a candidaturas) para conseguir representación parlamentaria.

\section{CUADRO 5}

CONOCIMIENTO DE LA EXISTENCIA DE UNA BARRERA LEGAL DEL 3 POR 100 EN EL SISTEMA ELECTORAL DE CATALUÑA, 2012*

\begin{tabular}{|l|c|c|c|}
\hline & Acierta & No acierta & No sabe \\
\hline Barrera legal & 28 & 8 & 65 \\
& $(277)$ & $(75)$ & $(642)$ \\
\hline
\end{tabular}

FUENTE: MEDW.

NOTA: * En cada casilla aparece, en primer lugar, el porcentaje de fila y, entre paréntesis, el número de individuos.

Las variables independientes recogen los determinantes de la atribución correcta de responsabilidades que se plantean en las hipótesis de la información y del interés, además de las preferencias sobre el modelo de organización territorial del Estado. Son las siguientes:

- Edad (en años);

- Nivel de renta mensual de todos los miembros del hogar de acuerdo con la siguiente escala: - Menos de 400 euros (1), - Entre 400 y 750 (2), - Entre 750 y 1250 (3), - Entre 1.250 y 1750 (4), - Entre 1.750 y 2500 (5), - Entre 2500 y 3250 (6), - Entre 3250 y 4000 (7), - Entre 4000 y 5000; (8), - Entre 5000 y 6500 (9), - Entre 6500 y 9000 (10), - Entre 9000 y 11000 (11), - Más de 11000 (12);

- Nivel de estudios más alto que se ha finalizado: - Sin finalizar la Educación Secundaria Obligatoria (1) - Educación Secundaria Obligatoria (ESO) (2), - Bachillerato o Formación Profesional de grado medio (3), - Diploma Universitario o Formación Profesional de grado superior (4), - Licenciatura Universitaria (5), - Estudios de posgrado (máster/doctorado) (6);

- Interés por la política en una escala de 0 (ninguno) a 10 (máximo);

- Votante (1) o no (0) en las elecciones autonómicas de Cataluña en 2012;

- Si el individuo se siente cercano a algún partido político (1) o no (0);

- Preferencias sobre el modelo de organización territorial de Estado: - Un Estado con un único Gobierno Central, sin comunidades autónomas (1), - Un Estado con comunidades autónomas pero con menos competencias que en la actuali- 
dad (2), - Un Estado con comunidades autónomas, como en la actualidad (3), - Un Estado con comunidades autónomas pero en el que éstas tengan más competencias que en la actualidad (4), - Un Estado federal, en el que las actuales comunidades autónomas tengan la condición de Estado pero siguiendo dentro de España (5) - Un Estado en el que sea posible que aquellas comunidades autónomas que lo quieran puedan convertirse en Estados independientes (6).

Los estadísticos descriptivos de las variables independientes se presentan en el cuadro $6^{11}$.

\section{CUADRO 6}

ESTADÍSTICOS DESCRIPTIVOS DE LAS VARIABLES INDEPENDIENTES

\begin{tabular}{|l|c|c|c|c|c|}
\hline \multicolumn{1}{|c|}{ Variables } & $\begin{array}{c}\text { Observa- } \\
\text { ciones }\end{array}$ & Media & $\begin{array}{c}\text { Desviación } \\
\text { típica }\end{array}$ & Mínima & Máxima \\
\hline Edad & 993 & 44,51 & 14,37 & 18 & 83 \\
\hline Nivel de renta & 880 & 4,81 & 1,94 & 1 & 12 \\
\hline Nivel de estudios & 993 & 3,46 & 1,09 & 1 & 6 \\
\hline Interés por la política & 991 & 6,11 & 2,83 & 0 & 10 \\
\hline Votante & 799 & 0,91 & 0,29 & 0 & 1 \\
\hline Cercanía partidos & 993 & 0,43 & 0,50 & 0 & 1 \\
\hline Modelo territorial & 931 & 4,73 & 1,61 & 1 & 6 \\
\hline
\end{tabular}

En definitiva, la especificación econométrica estimada es la siguiente:

$$
\begin{aligned}
& I_{i}=\beta_{0}+\beta_{1} \text { Edad }_{i}+\beta_{2} \text { Renta }_{i}+\beta_{3} \text { Estudios }_{i}+\beta_{4} \text { Interéspolítica }_{i}+ \\
& +\beta_{5} \text { Votante }_{i}+\beta_{6} \text { Cercaníapartidos }_{i}+\beta_{7} \text { Modeloterritorial }_{i}+\varepsilon_{i}
\end{aligned}
$$

donde el subíndice $i$ indica individuo e $I$ es, sucesivamente, el índice agregado y la respuesta a cada una de las cinco preguntas sobre atribución de competencias y sobre la barrera legal antes referidas.

Los resultados de las estimaciones se presentan en el Cuadro 7. En el modelo en que la variable dependiente es el índice agregado, estimado por MCO siguiendo a León $(2010,2012)^{12}$, la edad, el interés por la política y haber votado en las eleccio-

${ }^{11}$ La correlación más elevada entre las variables independientes, 0 ,29, se produce entre el nivel de renta y el de estudios.

Aunque un modelo logit o probit ordenado sería más adecuado para tratar el índice, hemos realizado una regresión lineal debido a que es lo que hace LEÓN (2010, 2012), Y como queremos mostrar los problemas que se derivan de usar el índice, respetamos la metodología que pone en práctica León. 
nes tienen un impacto positivo y estadísticamente significativo al 5 por 100 o mejor sobre la atribución correcta de responsabilidades políticas. Es decir, los individuos de más edad y más implicados en la política tienen un mejor conocimiento de la distribución de competencias entre el Gobierno central y el de la Generalitat. Además, los individuos que defienden el statu quo del modelo territorial del Estado distinguen peor entre los niveles de gobierno competentes en cada materia en comparación con los que prefieren un único Gobierno central sin Comunidades Autónomas. El resto de las variables no son estadísticamente significativas.

Sin embargo, cuando se desagrega el índice y se estima el modelo para cada una de las políticas, la principal conclusión es que el impacto de las variables no es robusto. La edad tiene un efecto positivo sobre la asignación correcta de las competencias en todas las políticas, pero no es estadísticamente significativa en el caso de las prestaciones por desempleo. Como se esperaba, el coeficiente de la variable es sensiblemente mayor cuando se trata de conocer quién decide sobre las pensiones y la sanidad. Se confirma así la hipótesis del interés. Las simulaciones que se realizan en el Gráfico 3 dejan bien claro que la edad importa más en la política de pensiones que en las de sanidad, educación y prestaciones por desempleo, en este orden. El nivel de renta tiene un efecto positivo y estadísticamente significativo sobre el conocimiento de qué gobierno es el responsable de sanidad y educación, negativo y estadísticamente significativo en el caso de las pensiones, y negativo y no significativo en cuanto a las pensiones. Estos resultados están en línea con los del reciente estudio de López Laborda y Rodrigo (2013).

Por su parte, el interés por la política y haber votado en las elecciones de 2012, que son las variables que mejor recogen la hipótesis de la información, tienen un impacto positivo en todos los temas, aunque solo son estadísticamente significativos para las prestaciones por desempleo y la sanidad, respectivamente. Tanto el nivel de estudios como la cercanía a los partidos no son estadísticamente significativos en ningún caso. Finalmente, todas las categorías de la variable que mide las preferencias sobre el modelo de organización territorial del Estado tienen los signos esperados, pero solo los defensores del statu quo y de un Estado federal conocen mejor de una manera estadísticamente significativa la distribución de competencias que los partidarios de la centralización en algunos temas.

Cuando se explica el conocimiento de la existencia de una barrera legal en el sistema electoral, los resultados son mucho más similares al modelo que tiene el índice como variable dependiente que a cualquiera de los demás. Es decir, la utilización del índice neutraliza en gran medida la hipótesis del interés y sesga los coeficientes. 


\section{CUADRO 7}

DETERMINANTES INDIVIDUALES DE LA ATRIBUCIÓN CORRECTA
DE RESPONSABILIDADES

\begin{tabular}{|c|c|c|c|c|c|c|}
\hline & \multicolumn{6}{|c|}{ Modelos } \\
\hline & $\begin{array}{c}\text { Índice } \\
\text { agregado }\end{array}$ & $\begin{array}{c}\text { Pensio- } \\
\text { nes }\end{array}$ & Sanidad & $\begin{array}{l}\text { Educa- } \\
\text { ción }\end{array}$ & $\begin{array}{c}\text { Desem- } \\
\text { pleo }\end{array}$ & $\begin{array}{c}\text { Barrera } \\
\text { legal }\end{array}$ \\
\hline Edad & $\begin{array}{c}0,014 * * * \\
(0,003)\end{array}$ & $\begin{array}{c}0,04 * * * \\
(0,01)\end{array}$ & $\begin{array}{c}0,03 * * * \\
(0,01)\end{array}$ & $\begin{array}{c}0,02 * * * \\
(0,01)\end{array}$ & $\begin{array}{c}0,01 \\
(0,01)\end{array}$ & $\begin{array}{c}0,014 * * \\
(0,006)\end{array}$ \\
\hline Nivel de renta & $\begin{array}{c}0,03 \\
(0,02)\end{array}$ & $\begin{array}{l}-0,09 \\
(0,07)\end{array}$ & $\begin{array}{c}0,18^{* * * *} \\
(0,05)\end{array}$ & $\begin{array}{c}0,13 * * * \\
(0,05)\end{array}$ & $\begin{array}{l}-0,11^{*} \\
(0,06)\end{array}$ & $\begin{array}{c}0,13 * * * \\
(0,05)\end{array}$ \\
\hline Nivel de estudios & $\begin{array}{c}0,03 \\
(0,04)\end{array}$ & $\begin{array}{c}0,12 \\
(0,12)\end{array}$ & $\begin{array}{c}0,01 \\
(0,09)\end{array}$ & $\begin{array}{l}-0,03 \\
(0.09)\end{array}$ & $\begin{array}{c}0,15 \\
(0,10)\end{array}$ & $\begin{array}{c}0,14 \\
(0,08)\end{array}$ \\
\hline Interés por la política & $\begin{array}{c}0,033 * * \\
(0,015)\end{array}$ & $\begin{array}{c}0,05 \\
(0,05)\end{array}$ & $\begin{array}{c}0,04 \\
(0,03)\end{array}$ & $\begin{array}{c}0,04 \\
(0,03)\end{array}$ & $\begin{array}{c}0,08 * * \\
(0,04)\end{array}$ & $\begin{array}{c}0,11^{* * *} \\
(0,04)\end{array}$ \\
\hline Votante & $\begin{array}{c}0,28 * * \\
(0,14)\end{array}$ & $\begin{array}{c}0,33 \\
(0,38)\end{array}$ & $\begin{array}{c}0,78 * * * \\
(0,29)\end{array}$ & $\begin{array}{c}0,25 \\
(0,43)\end{array}$ & $\begin{array}{c}0,09 \\
(0,35)\end{array}$ & $\begin{array}{l}-0,39 \\
(0,31)\end{array}$ \\
\hline Cercanía partidos & $\begin{array}{c}0,10 \\
(0,09)\end{array}$ & $\begin{array}{c}0,38 \\
(0,28) \\
\end{array}$ & $\begin{array}{l}-0,02 \\
(0,20) \\
\end{array}$ & $\begin{array}{c}0,12 \\
(0,19) \\
\end{array}$ & $\begin{array}{c}0,31 \\
(0,23) \\
\end{array}$ & $\begin{array}{c}0,58 * * * \\
(0,19)\end{array}$ \\
\hline \multicolumn{7}{|c|}{$\begin{array}{l}\text { Modelo territorial } \\
\text { (ref.) Un único Gobierno Central, sin CCAA }\end{array}$} \\
\hline $\begin{array}{l}\text { CCAA con menos compe- } \\
\text { tencias }\end{array}$ & $\begin{array}{c}0,20 \\
(0,21)\end{array}$ & $\begin{array}{c}0,69 \\
(0,68)\end{array}$ & $\begin{array}{c}0,15 \\
(0,43)\end{array}$ & $\begin{array}{c}0,26 \\
(0,43)\end{array}$ & $\begin{array}{c}0,28 \\
(0,53)\end{array}$ & $\begin{array}{l}-0,45 \\
(0,47)\end{array}$ \\
\hline Como en la actualidad & $\begin{array}{c}-0,58^{* *} \\
(0,27)\end{array}$ & $\begin{array}{l}-0,44 \\
(0,65)\end{array}$ & $\begin{array}{l}-1,00^{*} \\
(0,57)\end{array}$ & $\begin{array}{l}-1,01 * \\
(0,56)\end{array}$ & $\begin{array}{l}-0,19 \\
(0,62)\end{array}$ & $\begin{array}{c}0,42 \\
(0,58)\end{array}$ \\
\hline CCAA con más competencias & $\begin{array}{c}0,29 \\
(0,19)\end{array}$ & $\begin{array}{c}0,83 \\
(0,64)\end{array}$ & $\begin{array}{c}0,43 \\
(0,40)\end{array}$ & $\begin{array}{c}0,21 \\
(0,39)\end{array}$ & $\begin{array}{c}0,64 \\
(0,53)\end{array}$ & $\begin{array}{c}0,15 \\
(0,42)\end{array}$ \\
\hline Estado federal & $\begin{array}{c}0,27 \\
(0,16)\end{array}$ & $\begin{array}{c}0,27 \\
(0,46)\end{array}$ & $\begin{array}{l}0,59 * \\
(0,34)\end{array}$ & $\begin{array}{c}0,52 \\
(0,34)\end{array}$ & $\begin{array}{c}0.07 \\
(0,40)\end{array}$ & $\begin{array}{l}-0,32 \\
(0,35)\end{array}$ \\
\hline $\begin{array}{l}\text { CCAA puedan ser indepen- } \\
\text { dientes }\end{array}$ & $\begin{array}{c}0,14 \\
(0.16)\end{array}$ & $\begin{array}{l}-0,12 \\
(0,44)\end{array}$ & $\begin{array}{c}0,48 \\
(0,33)\end{array}$ & $\begin{array}{c}0,34 \\
(0,32)\end{array}$ & $\begin{array}{l}-0,13 \\
(0,38)\end{array}$ & $\begin{array}{l}-0,06 \\
(0,35)\end{array}$ \\
\hline Constante & $\begin{array}{c}1,55^{* * * *} \\
(0,25)\end{array}$ & $\begin{array}{l}-0,18 \\
(0,73)\end{array}$ & $\begin{array}{c}-2,51 * * * \\
(0,57)\end{array}$ & $\begin{array}{c}-1,47 * * * \\
(0,55)\end{array}$ & $\begin{array}{c}0,31 \\
(0,63)\end{array}$ & $\begin{array}{c}-3,11 * * * \\
(0,58)\end{array}$ \\
\hline R2 / Pseudo R2 & 0,10 & 0,06 & 0,09 & 0,06 & 0,03 & 0,07 \\
\hline$\%$ casos correctamente predichos & - & 88,08 & 72,43 & 71,09 & 82,77 & 69,90 \\
\hline $\mathrm{N}$ & 669 & 669 & 669 & 669 & 669 & 669 \\
\hline Estimación & $\mathrm{MCO}$ & Logit & Logit & Logit & Logit & Logit \\
\hline
\end{tabular}

NOTA: En cada casilla aparece, en primer lugar, el coeficiente de regresión, seguido del error típico, entre paréntesis. * $\mathrm{p}>0,1 ; * * \mathrm{p}<0,05 ; * * * \mathrm{p}<0,01$. 

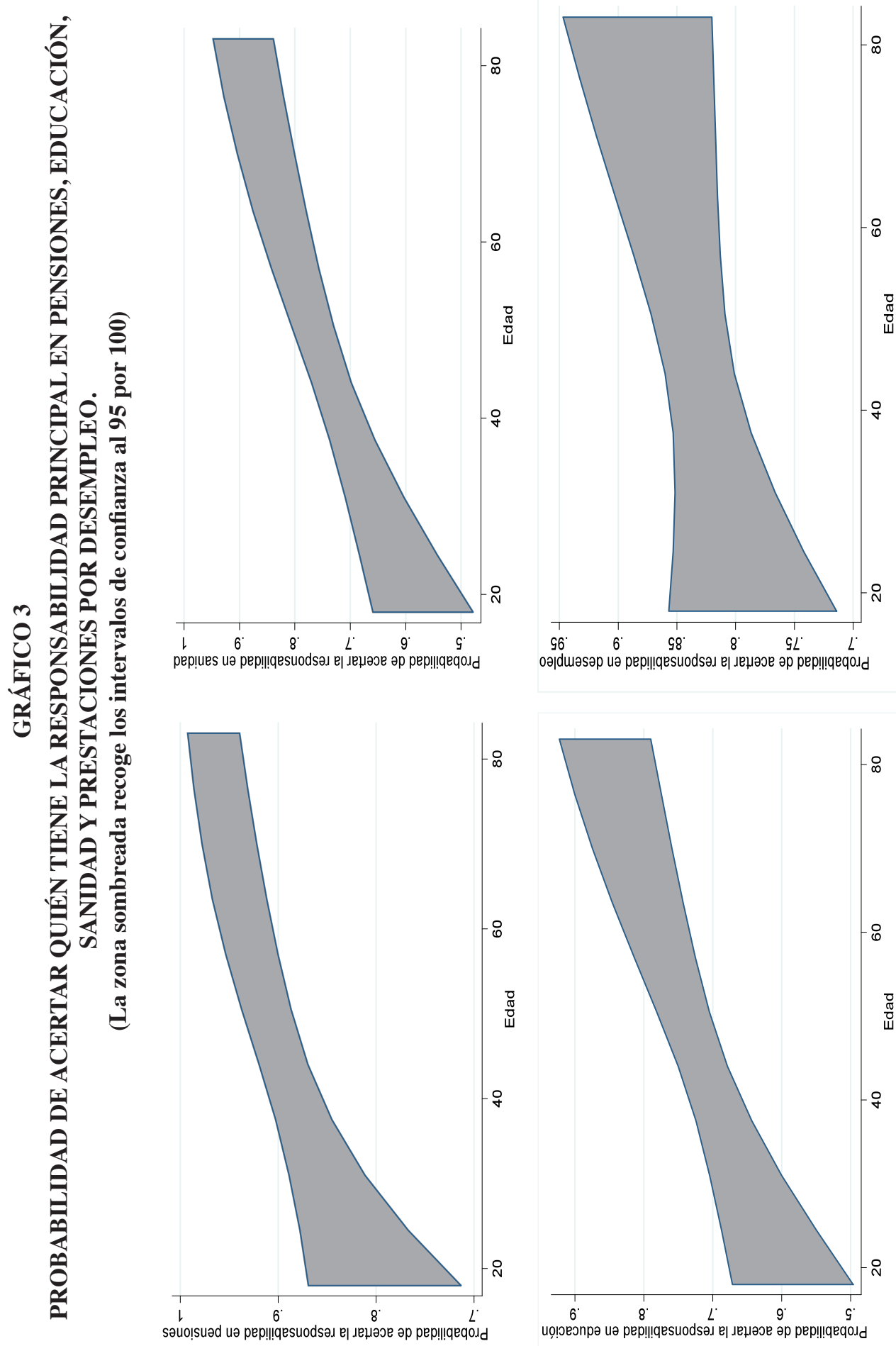


\section{Conclusiones}

La atribución correcta de las responsabilidades políticas es imprescindible para que el control electoral de los gobiernos sea efectivo. Si los ciudadanos no saben cuál es el grado de responsabilidad que cabe atribuir a un gobierno en los resultados económicos, ya sean buenos o malos, difícilmente pueden castigar a los malos gobiernos y recompensar a los buenos. En este artículo, basado en datos individuales sobre el Estado de las Autonomías en España, se han establecido tres conclusiones. En primer lugar, la asignación de responsabilidades es contingente de los arreglos institucionales, en el sentido de que las reglas de juego aumentan o atenúan la capacidad de los ciudadanos para saber en manos de quién están las políticas que se ponen en marcha. Cuando las competencias están en manos del gobierno nacional, el acierto de los ciudadanos en la imputación de la responsabilidad política es mayor que cuando los gobiernos autonómicos son los titulares. Por ejemplo, más de la mitad de los españoles asigna incorrectamente la responsabilidad en sanidad o educación, mientras que menos de un tercio se equivoca en el caso de las pensiones de jubilación o el seguro por desempleo. Los resultados son similares en los impuestos. De todos modos, de acuerdo con la evidencia que tenemos, este acierto es mayor en las Comunidades Autónomas que más tiempo han disfrutado de competencias propias.

En segundo lugar, desde un punto de vista dinámico, los últimos datos disponibles, correspondientes a 2010, reflejan máximos históricos en el porcentaje de aciertos en la asignación de responsabilidades. A expensas de lo que diga la evidencia empírica, nuestra hipótesis tentativa es que la recesión económica, los recortes derivados de la consolidación fiscal y la máxima relevancia de las cuestiones económicas y fiscales en los últimos años podrían haber tenido como efecto positivo no intencionado la mejora de la comprensión del sistema de atribución de competencias. Se trata de una cuestión abierta para la investigación futura.

Finalmente, cuando se explican las diferencias entre los individuos a la hora de atribuir correctamente las responsabilidades políticas, hemos distinguido las hipótesis de la información y del interés. Las dos hipótesis no van siempre en la misma dirección, de modo que los efectos de los determinantes individuales varían según cuál sea la política. La edad es la variable que tiene el efecto más sistemático. De este modo, la creación de índices que agregan el conocimiento sobre quién tiene la responsabilidad acerca de distintos temas sesga los resultados a favor de la hipótesis de la información.

\section{Referencias bibliográficas}

[1] AIDT, T. S. (2000): «Economic voting and information», Electoral Studies, 19: 349-362.

[2] ANDERSON, C. A. (2006): «Economic Voting and Multilevel Governance: A Comparative Individual-Level Analysis», American Journal of Political Science 50: 449463. 
[3] BANCO MUNDIAL (2000): The World Development Report 1999/2000. Entering the $21^{\text {st }}$ Century. Washington, D.C.: The World Bank and Oxford: Oxford University Press.

[4] BLAIS, A. (2010): «Making Electoral emocracy Work», Electoral Studies, 29, 169-170.

[5] CUTLER, F. (2004): «Government Responsibility and Electoral Accountability in Federations», Publius. The Journal of Federalism, 34: 19-38.

[6] DUCH, R. M.; PALMER, H. D. y ANDERSON, C. J. (2000): «Heterogeneity in Perceptions of National Economic Conditions», American Journal of Political Science, 44: 635-652.

[7] FERNÁNDEZ LLERA, R. y LAGO PEÑAS, S. (2011): «Ultraperiferia, economía y finanzas públicas de Canarias”, Revista de Estudios Regionales, 90: 17-44.

[8] GIL-RUIZ, C. L. e IGLESIAS, J. (2007): «El gasto público en España en un contexto descentralizado», Presupuesto y Gasto Público, 47: 185-206.

[9] IEF (2006): «Opiniones y actitudes fiscales de los españoles en 2005». Documentos de trabajo del Instituto de Estudios Fiscales, 6/10. (http://www.ief.es/documentos/recursos/publicaciones/documentos_trabajo/2006_10.pdf).

[10] IEF (2011): «Opiniones y actitudes fiscales de los españoles en 2010». Documentos de trabajo del Instituto de Estudios Fiscales, 9/11. (http://www.ief.es/documentos/recursos/publicaciones/documentos_trabajo/2011_09.pdf).

[11] LAGO PEÑAS, I. y LAGO PEÑAS, S. (2010): «Descentralization and Electoral Accountability», Environment and Policy, 28, 318-334.

[12] LAGO PEÑAS, I. y LAGO PEÑAS, S. (2011): Descentralización y control electoral de los gobiernos en España. Barcelona: Institut d'Estudis Autonòmics.

[13] LAGO PEÑAS, S. y MARTÍNEZ-VÁZQUEZ, J. (eds.) (2009): La asignación de impuestos a las CC.AA.: desafios y oportunidades. Madrid: Instituto de Estudios Fiscales.

[14] LAGO PEÑAS, S. y MARTÍNEZ-VÁZQUEZ, J. (eds.) (2011): Las transferencias intergubernamentales en España: análisis y propuestas de reforma. Madrid: Instituto de Estudios Fiscales.

[15] LEÓN, S. (2010): «Who is responsible for what? Clarity of responsibilities in multilevel states: The case of Spain», European Journal of Political Research, 50: 80-109.

[16] LEÓN, S. (2012): «How do citizens attribute responsibility in multilevel states? Learning, biases and asymmetric federalism. Evidence from Spain», Electoral Studies, 31: 120-130.

[17] LÓPEZ LABORDA, J. y RODRIGO, F. (2013): «Nueva evidencia sobre atribución de responsabilidades sobre los servicios regionales y preferencia por la descentralización», Papeles de Trabajo del IEF, 3/2013.

[18] McGRAW, K. M. (1990): «Avoiding Blame: An Experimental Investigation of Political Excuses and Justifications», British Journal of Political Science, 20: 119-131.

[19] O’NEILL, K. (2003): «Decentralization as a Political Strategy», Comparative Political Studies, 26: 1068-1091.

[20] POWELL, G. B. Jr. y WHITTEN, G. (1993): «A cross-national analysis of economic voting: Taking account of the political context», American Journal of Political Science, 37: 391-414.

[21] PRZEWORSKI, A.; STOKES, S. C. y MANIN, B. (1999) (eds.): Democracy, accountability and representation. Nueva York: Cambridge University Press. 
[22] RUDOLPH, T. J. (2003a): «Institutional Context and the Assignment of Political Responsibility», Journal of Politics, 65: 190-215.

[23] RUDOLPH, T. J. (2003b): «Who's Responsible for the Economy? The Formation and Consequences of Responsibility Attributions», American Journal of Political Science, 47: 698-713. 